\title{
Sample size calculation on web, can we rely on the results?
}

\author{
Alipasha Meysamie ${ }^{1 *}$, Farough Taee ${ }^{1}$, Mohammad-Ali Mohammadi-Vajari', Siamak Yoosefi-Khanghah', Sahra Emamzadeh-Fard ${ }^{1}$ and \\ Mehrshad Abbassi ${ }^{2}$ \\ *Correspondence: Meysamie@tums.ac.ir \\ 'Department of Community and Preventive Medicine, Medical Faculty, Tehran University of Medical Sciences, Iran. \\ ${ }^{2}$ Department of Nuclear Medicine, Valiasr Hospital, Tehran University of Medical Sciences, Tehran, Iran.
}

\begin{abstract}
One of the most frequent concerns of the researches is sample size calculation. There are many equations to calculate sample size one should be selected correctly based on the type of the study and data type. Any mistake can mislead the researcher and so the study results. Even an overestimation can cause waste of cost, time and material. Either statisticians or other researchers frequently use online advice on sample size calculation. Online sample size calculators have been searched, reviewed and checked for the calculated results based on known formulas for common research objectives. Considering the most common types of health study objectives regarding sample size calculation (estimating proportion or mean, comparison of two proportions or two means), from 60 website or software, only 5(8.3\%) provided all four types of calculations. Overall exact calculation rate was $8.3 \%$ and $16.1 \%$ per calculation and per site, respectively. Many of the sites just calculate sample size for estimating proportions and most of the results lack the accuracy in calculation.
\end{abstract}

Keywords: Online calculation, sample size, health studies, accuracy

\section{Introduction}

The statisticians, methodologists and non-statistician professional researchers frequently ask this question "how many subjects do I need for my study?" [1]. The sample size affect the feasibility of the research and proper calculation possess significant effect on the power and validity of the results [2]. Currently, the researchers solve most of their problems via online solutions and face to face consultations are limited for the must to do occasions. For internet assisted sample size calculation, the first step is to find a proper site with online calculation capability or downloadable files but the accuracy of calculations is one of the most important matters. There are many online sample size calculation sites with different specifications. Non-statistician users do not like sophisticated processes for calculation and more professional statisticians may need advanced calculations for somehow rare study types. In this review the sample size calculation websites have been listed and tried to be checked how their calculations are the same as basic formulas show.

\section{Methods}

Google search engine have been used with keywords including sample size calculator, sample size calculation, sample size in health studies, sample size web site and sample size software for retrieving related sites. Software informer website (available at http://software.informer.com) also used to find downloadable files for sample size calculation. Each site reviewed and data concerning URL, owner, availability of calculation equation(s), providing other types of calculations rather than only sample size calculation e.g. confidence levels or statistical tests collected. Standard formulas for sample size calculation extracted considering the four most common types of sample size calculation in health studies for estimating proportion of a qualitative variable, a mean of a quantitative variable and comparison of two proportions and two means of two different populations according to detailed review of several statistical resources, such as some biostatistics textbooks [3-8] and articles [9]. Four hypothetical examples considered for calculations (one for each) and the results based on selected formulas calculated (Table 1). Then the results of each site or software compared with the standard results. A difference more than rounding the final results (more than 1) with the standard result, considered as inaccurate (not the same as formula) calculation. The feasibility of site usage also evaluated to determine whether the application of sites was simple and user friendly or professional. To evaluate the complexity, two medical researchers who had some experience in medical research and sample size calculation asked to use sample size calculators to score them as easy, moderate or advanced to use. The sites scored as easy if they required basic input data for calculation and instructed each phrase of calculation clearly for users; the sites which needed detailed information about the study e.g. one tailed or two tailed calculations considered as moderate; and the sites which required very detailed and additional information which were not familiar to routine practices in medical research according to the basis of statistical knowledge as a medical researchers e.g. complex survey analysis or cost analysis considered as advanced. The congruent rating of both observers for sites considered as the ease of use level and if there was any incongruence between their assessment, a third evaluator considered the site review for rating. 
Meysamie et al. Journal of Medical Statistics and Informatics 2014,

http://www.hoajonline.com/journals/pdf/2053-7662-2-3.pdf

doi: $10.7243 / 2053-7662-2-3$

Table 1. Different types of formula for sample size calculation and hypothetical data and results used for assessing the calculation accuracy ( $\mathrm{z}=$ value on standardized normal distribution for giving probability).

\begin{tabular}{|c|c|c|c|}
\hline Type of study & Formula & Hypothetical used data & $\begin{array}{l}\text { Calculated result } \\
\text { based on formula }\end{array}$ \\
\hline $\begin{array}{l}\text { Estimation of a } \\
\text { proportion }\end{array}$ & $n=\frac{z_{1-\frac{\alpha}{2}}^{2} p q}{d^{2}}$ & $\begin{array}{l}\mathrm{p}=\text { Given prevalence }=40 \% \\
\quad \alpha=\text { Type } \mathrm{I} \text { error }=5 \% \\
\mathrm{~d}=\text { Acceptable difference from prevalence }=5 \%\end{array}$ & 369 \\
\hline $\begin{array}{l}\text { Estimation of a } \\
\text { mean }\end{array}$ & $n=\frac{{ }_{1-\frac{\alpha}{2}}^{2} \sigma^{2}}{d^{2}}$ & $\begin{array}{l}\sigma=\text { Standard Deviation }=10 \\
\quad \alpha=\text { Type I error }=5 \% \\
d=\text { Acceptable difference from mean }=3\end{array}$ & 43 \\
\hline $\begin{array}{l}\text { Comparison of two } \\
\text { proportion }\end{array}$ & $n=\frac{2\left(z_{1-\frac{\alpha}{2}}+z_{1-\beta}\right)^{2} \hat{p} \hat{q}}{\left(p_{1}-p_{2}\right)^{2}}$ & $\begin{array}{c}\alpha=\text { Type I error }=5 \% \\
\beta=20 \% \\
\mathrm{p}_{1}=\text { Given prevalence for group } 1=40 \% \\
\mathrm{p}_{2}=\text { Given prevalence for group } 2=60 \%\end{array}$ & 99 for each group \\
\hline $\begin{array}{l}\text { Comparison of two } \\
\text { mean }\end{array}$ & $n=\frac{\left(z_{1-\frac{\alpha}{2}}+z_{1-\beta}\right)^{2}\left(\sigma_{1}^{2}+\sigma_{2}^{2}\right)}{\left(\mu_{1}-\mu_{2}\right)^{2}}$ & $\begin{array}{l}\alpha=\text { Type I error }=5 \% \\
\beta=\text { Type II error }=20 \% \\
\sigma_{1}=\text { Standard Deviation for group } 1=7 \\
\sigma_{2}=\text { Standard Deviation for group } 2=8 \\
\mu_{1}=\text { mean for group } 1=25 \\
\mu_{2}=\text { mean for group } 2=22\end{array}$ & 99 for each group \\
\hline
\end{tabular}

Two sites could not be rated because of unavailability of registration or access for authors $[11,52]$ and some had no calculation regarding to the four common ways considered in evaluation process $[12,17,18,27,31,34,52,55,57]$ (Table 2). STATA software also used for calculations and related results depicted [59].

\section{Results}

The summary of available sample size calculator sites is presented in (Table 2). Among the web-based sample size calculators, some do the statistics online and other provides a downloadable file for calculations $[10,11,21,22,24,26,34,53$, $57,58]$. These files vary from a static table in excel [21] or dynamic file for multiple calculations [10]. Most of the sites (25 out of 50) just calculate sample size for estimation of a proportion, and most of them consider a proportion of $50 \%$ as a fixed number in formula for calculation and so the results were not accurate for considered example in the study. Only five sites provided all four common types of calculations $[16,23,33,45,46]$. Considering calculations in comparison with the results of formulas, from 57 different calculations, $18(31.6 \%)$ were accurate and based on the sites, only $13(26.0 \%)$ sites provides at least one accurate result. From those providing all four types of calculations only one had accurate results for all four calculations based on formulas [23] and the other one [16] provided accurate results for 3 calculations. Some sites provided calculations for specific studies e.g. complex sample surveys $[49,57]$, survival analysis $[26,55]$ and microarray experiments in genetic studies [12] and some provided multi language support $[23,37]$. From 48 rated sites $28(58.3 \%)$ scored as "Easy", 6(14.6\%) as "Moderate" and 10(27.1\%) as "Advanced" according to the complexity of the site usage.

\section{Discussion}

We provided a list of available online sample size calculating sites with brief description and an evaluation of their accuracy in calculations, besides the level of ease of use and complexity.

Online sample size calculators vary from easy and userfriend to advanced and professional, but what is at the most important matter is "How valid are their calculations?". According to our findings, there are few sites which provides all common ways of sample size calculation needed for common types of researches and only a few provides accurate calculations. The most important reason for inaccurate results may be due to some fix considerations in many sites e.g. considering a fix proportion of $50 \%$ for calculation of sample size in studies when the aim is estimation of prevalence. Some other reasons may be different formulas used in sites rather those we considered in our assessment. It seems providing basic formulas for each calculation, may help researchers to choose more accurate path for sample size calculation. Although in some conditions e.g. comparison of two proportions different formulas will provide almost the same results for sample size calculation. In other conditions like estimation there are somehow more different formulas for sample size calculations and so more apart results. This has been shown in our study that some sites used the formula which considered type II error for sample size calculation for estimation a proportion or a mean [33]. Some sites may use more accurate formulas for calculation but others use inaccurate formulas. For example some sites considers formulas using just one standard deviation for calculation in case of two mean comparison $[24,26,46]$ 
Table 2. Brief description of sample size calculator websites and software, NR=Not Rated, NAP=Not Applicable, NC=No calculation provided.

\begin{tabular}{|c|c|c|c|c|c|c|c|c|c|}
\hline \multirow{3}{*}{ 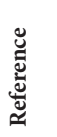 } & \multirow{3}{*}{$\begin{array}{l}\text { Providing } \\
\text { Formulas }\end{array}$} & \multirow[t]{3}{*}{ Usability } & \multirow{3}{*}{$\begin{array}{l}\text { Downloadable } \\
\text { File(s) }\end{array}$} & \multirow{3}{*}{$\begin{array}{l}\text { Type of Calculations } \\
\text { Provided }\end{array}$} & \multirow{3}{*}{$\begin{array}{l}\text { Accurate } \\
\text { calculation }\end{array}$} & \multicolumn{4}{|c|}{ Common Sample Size Calculation Results } \\
\hline & & & & & & \multicolumn{2}{|l|}{ Estimation } & \multicolumn{2}{|l|}{ Comparison } \\
\hline & & & & & & Qualitative $^{1}$ & Quantitative $^{2}$ & Qualitative $^{3}$ & Quantitative $^{4}$ \\
\hline$[10]$ & No & Advanced & $\begin{array}{l}\text { Yes } \\
\text { (An excel file) }\end{array}$ & $\begin{array}{l}\text { Calculates one sample mean } \\
\text { estimation sample size and } \\
\text { also power, error and cost }\end{array}$ & No & $\mathrm{NC}$ & $\mathrm{NC}$ & $\mathrm{NC}$ & $\mathrm{NC}$ \\
\hline$[11]$ & Yes & NR & Yes & $\begin{array}{l}\text { Needs registration for } \\
\text { calculation or file } \\
\text { download }\end{array}$ & Not Rated & - & - & - & - \\
\hline$[12]$ & No & Easy & No & $\begin{array}{l}\text { Calculates sample size for } \\
\text { microarray experiments in } \\
\text { genetic studies }\end{array}$ & NAP & $\mathrm{NC}$ & NC & NC & NC \\
\hline$[13]$ & Yes & Easy & No & $\begin{array}{l}\text { Only for } 50 \% \text { estimate } \\
\text { Considers population size } \\
\text { Calculates Confidence } \\
\text { intervals }\end{array}$ & No & 383 & NC & $\mathrm{NC}$ & NC \\
\hline$[14]$ & Yes & Moderate & $\begin{array}{l}\text { Yes } \\
\text { (Also online) }\end{array}$ & $\begin{array}{l}\text { Only for } 50 \% \text { estimate } \\
\text { Considers population size } \\
\text { Calculates Confidence } \\
\text { intervals }\end{array}$ & No & 383 & NC & NC & NC \\
\hline$[15]$ & Yes & Easy & $\begin{array}{l}\text { Yes } \\
\text { (Also online) }\end{array}$ & $\begin{array}{l}\text { Only for } 50 \% \text { estimate } \\
\text { Considers population size }\end{array}$ & No & 383 & $\mathrm{NC}$ & $\mathrm{NC}$ & $\mathrm{NC}$ \\
\hline$[16]$ & No & Moderate & No & $\begin{array}{l}\text { Provides all } 4 \text { types of sample } \\
\text { size calculations } \\
\text { Considers population size } \\
\text { One and two tailed } \\
\text { calculations }\end{array}$ & $\begin{array}{l}\text { Yes } \\
\text { (just 1,2\&4) }\end{array}$ & 369 & 43 & $107+107$ & $\begin{array}{l}99+99 \\
\text { Uses } \\
\text { Variances }\end{array}$ \\
\hline$[17]$ & No & Advanced & No & $\begin{array}{l}\text { Provides statistical tests for } \\
\text { different types of RCT } \\
\text { No sample size calculation }\end{array}$ & NC & $\mathrm{NC}$ & $\mathrm{NC}$ & $\mathrm{NC}$ & $\mathrm{NC}$ \\
\hline$[18]$ & No & Easy & No & $\begin{array}{l}\text { Provides Java applets for Dif- } \\
\text { ferent statistical tests and con- } \\
\text { fidence interval calculations } \\
\text { No sample size calculation }\end{array}$ & NAP & $\mathrm{NC}$ & $\mathrm{NC}$ & $\mathrm{NC}$ & $\mathrm{NC}$ \\
\hline$[19]$ & No & Easy & No & $\begin{array}{l}\text { Just for a qualitative } \\
\text { estimation } \\
\text { Considers population size } \\
\text { Calculates Confidence } \\
\text { intervals } \\
\text { Calculates Confidence } \\
\text { intervals }\end{array}$ & Yes & 367 & NC & $\mathrm{NC}$ & $\mathrm{NC}$ \\
\hline$[20]$ & Yes & Easy & No & $\begin{array}{l}\text { Only for } 50 \% \text { estimate } \\
\text { Considers population size } \\
\text { Calculates Confidence } \\
\text { intervals }\end{array}$ & No & 382.6937 & NC & $\mathrm{NC}$ & NC \\
\hline$[21]$ & No & Moderate & $\begin{array}{l}\text { Yes } \\
\text { (An excel file) }\end{array}$ & $\begin{array}{l}\text { Provides No calculation } \\
\text { Table is Only for } 50 \% \text { estimate }\end{array}$ & No & $\mathrm{NC}$ & $\mathrm{NC}$ & NC & NC \\
\hline$[22]$ & No & Easy & Yes & Provides only a monogram & No & 383 & NC & NC & NC \\
\hline$[23]$ & Yes & Easy & No & $\begin{array}{l}\text { Multilingual (Persian, } \\
\text { Arabic, French and English) } \\
\text { Provides all } 4 \text { types of sample } \\
\text { size } \\
\text { calculations } \\
\text { Calculates for non equal groups } \\
\text { Provides calculations for } \\
\text { correlation estimates }\end{array}$ & Yes & 369 & 43 & $99+99$ & $99+99$ \\
\hline
\end{tabular}


Meysamie et al. Journal of Medical Statistics and Informatics 2014,

continuation of Table 2

\begin{tabular}{|c|c|c|c|c|c|c|c|c|c|}
\hline \multirow{3}{*}{ 芯 } & \multirow{3}{*}{$\begin{array}{l}\text { Providing } \\
\text { Formulas }\end{array}$} & \multirow[t]{3}{*}{ Usability } & \multirow{3}{*}{$\begin{array}{l}\text { Downloadable } \\
\text { File(s) }\end{array}$} & \multirow{3}{*}{$\begin{array}{l}\text { Type of Calculations } \\
\text { Provided }\end{array}$} & \multirow{3}{*}{$\begin{array}{l}\text { Accurate } \\
\text { calculation }^{*}\end{array}$} & \multicolumn{4}{|c|}{ Common Sample Size Calculation Results } \\
\hline & & & & & & \multicolumn{2}{|l|}{ Estimation } & \multicolumn{2}{|l|}{ Comparison } \\
\hline & & & & & & Qualitative $^{1}$ & Quantitative $^{2}$ & Qualitative $^{3}$ & Quantitative $^{4}$ \\
\hline$[24]$ & No & Easy & Yes & $\begin{array}{l}\text { Provides PS: Power and } \\
\text { Sample Size Calculation } \\
\text { version } 3.0 \text { Software for } \\
\text { download } \\
\text { For windows, Macintosh } \\
\text { and Linux. } \\
\text { Calculates sample size and } \\
\text { power }\end{array}$ & No & $\mathrm{NC}$ & $\mathrm{NC}$ & $\mathrm{NC}$ & $\begin{array}{l}28+28 \\
\text { Considering } \\
\text { One SD }\end{array}$ \\
\hline$[25]$ & No & Easy & No & $\begin{array}{l}\text { Only for } 50 \% \text { estimate } \\
\text { Considers population size }\end{array}$ & No & 383 & $\mathrm{NC}$ & $\mathrm{NC}$ & $\mathrm{NC}$ \\
\hline$[26]$ & No & Advanced & $\begin{array}{l}\text { Yes } \\
\text { (A .jar file) }\end{array}$ & $\begin{array}{l}\text { Calculates sample size for } \\
\text { comparison of qualitative } \\
\text { and quantitative outcomes } \\
\text { and for survival data }\end{array}$ & Yes (just 3) & $\mathrm{NC}$ & $\mathrm{NC}$ & $\begin{array}{l}198 \\
\text { Total }\end{array}$ & $\begin{array}{l}504 \\
\text { Total } \\
\text { Considering } \\
\text { One SD }\end{array}$ \\
\hline$[27]$ & No & Moderate & No & $\begin{array}{l}\text { Calculates sample size for } \\
\text { usability studies. }\end{array}$ & NAP & $\mathrm{NC}$ & $\mathrm{NC}$ & $\mathrm{NC}$ & $\mathrm{NC}$ \\
\hline$[28]$ & No & Easy & No & $\begin{array}{l}\text { Only for } 50 \% \text { estimate } \\
\text { Considers population size }\end{array}$ & No & 383 & $\mathrm{NC}$ & $\mathrm{NC}$ & NC \\
\hline [29] & Yes & Advanced & No & $\begin{array}{l}\text { Just sample size } \\
\text { calculation for two } \\
\text { parallel samples in } \\
\text { comparison of } \\
\text { proportions } \\
\text { Provides Non equal sample } \\
\text { size calculation }\end{array}$ & No & $\mathrm{NC}$ & $\mathrm{NC}$ & 10 & $\mathrm{NC}$ \\
\hline$[30]$ & No & Easy & No & $\begin{array}{l}\text { A survey sample size } \\
\text { calculator } \\
\text { Only for } 50 \% \text { estimate } \\
\text { Considers population size } \\
\text { Calculates Confidence } \\
\text { intervals }\end{array}$ & No & 383 & $\mathrm{NC}$ & $\mathrm{NC}$ & NC \\
\hline$[31]$ & No & Advanced & No & $\begin{array}{l}\text { Calculates power and } \\
\text { Sample Size for Logistic } \\
\text { Regression with Binary } \\
\text { Covariates }\end{array}$ & NAP & $\mathrm{NC}$ & $\mathrm{NC}$ & $\mathrm{NC}$ & $\mathrm{NC}$ \\
\hline$[32]$ & No & Easy & No & $\begin{array}{l}\text { Only for } 50 \% \text { estimate } \\
\text { Considers population size }\end{array}$ & No & 383 & $\mathrm{NC}$ & NC & NC \\
\hline$[33]$ & No & Moderate & No & $\begin{array}{l}\text { Provides all } 4 \text { types of } \\
\text { sample size } \\
\text { calculations }\end{array}$ & No & $\begin{array}{l}606 \\
\text { With Beta } \\
\text { Error }\end{array}$ & $\begin{array}{l}69 \\
\text { With Beta } \\
\text { Error }\end{array}$ & $78+78$ & $76+76$ \\
\hline$[34]$ & No & Advanced & Yes & $\begin{array}{l}\text { Just provides links to } \\
\text { other pages } \\
\text { don't provide any direct } \\
\text { calculation }\end{array}$ & - & $\mathrm{NC}$ & $\mathrm{NC}$ & NC & $\mathrm{NC}$ \\
\hline$[35]$ & No & Easy & No & $\begin{array}{l}\text { Only for } 50 \% \text { estimate } \\
\text { Considers population size }\end{array}$ & No & 383 & $\mathrm{NC}$ & $\mathrm{NC}$ & $\mathrm{NC}$ \\
\hline$[36]$ & No & Easy & No & $\begin{array}{l}\text { Only for } 50 \% \text { estimate } \\
\text { Considers population size } \\
\text { Just for } 95 \% \text { and } 99 \% \\
\text { confidence }\end{array}$ & No & 383 & $\mathrm{NC}$ & $\mathrm{NC}$ & $\mathrm{NC}$ \\
\hline$[37]$ & No & Easy & No & $\begin{array}{l}\text { Multilingual (Chinese, } \\
\text { English, French, } \\
\text { Korean, Italian, } \\
\text { Japanese, Spanish) } \\
\text { Only for } 50 \% \text { estimate } \\
\text { Considers population size } \\
\text { Calculates Confidence } \\
\text { intervals }\end{array}$ & No & 383 & $\mathrm{NC}$ & $\mathrm{NC}$ & $\mathrm{NC}$ \\
\hline
\end{tabular}


continuation of Table 2.

\begin{tabular}{|c|c|c|c|c|c|c|c|c|c|}
\hline \multirow{3}{*}{ 苂 } & \multirow{3}{*}{$\begin{array}{l}\text { Providing } \\
\text { Formulas }\end{array}$} & \multirow[t]{3}{*}{ Usability } & \multirow{3}{*}{$\begin{array}{l}\text { Downloadable } \\
\text { File(s) }\end{array}$} & \multirow{3}{*}{$\begin{array}{l}\text { Type of Calculations } \\
\text { Provided }\end{array}$} & \multirow{3}{*}{$\begin{array}{l}\text { Accurate } \\
\text { calculation }^{\star}\end{array}$} & \multicolumn{4}{|c|}{ Common Sample Size Calculation Results } \\
\hline & & & & & & \multicolumn{2}{|l|}{ Estimation } & \multicolumn{2}{|l|}{ Comparison } \\
\hline & & & & & & Qualitative $^{1}$ & Quantitative $^{2}$ & Qualitative $^{3}$ & Quantitative $^{4}$ \\
\hline$[38]$ & No & Easy & No & $\begin{array}{l}\text { Only for } 50 \% \text { estimate } \\
\text { Considers population size } \\
\text { Calculates Confidence } \\
\text { intervals }\end{array}$ & No & 383 & $\mathrm{NC}$ & NC & NC \\
\hline$[39]$ & No & Easy & No & $\begin{array}{l}\text { Only for } 50 \% \text { estimate } \\
\text { Considers population size }\end{array}$ & No & 383 & $\mathrm{NC}$ & $\mathrm{NC}$ & $\mathrm{NC}$ \\
\hline$[40]$ & Yes & Easy & $\begin{array}{l}\text { Yes } \\
\text { (Also online) }\end{array}$ & $\begin{array}{l}\text { Only for } 50 \% \text { estimate } \\
\text { Considers population size } \\
\text { Calculates Confidence } \\
\text { intervals }\end{array}$ & No & 322 & $\mathrm{NC}$ & $\mathrm{NC}$ & $\mathrm{NC}$ \\
\hline$[41]$ & No & Easy & No & $\begin{array}{l}\text { Considers population size } \\
\text { Calculates Confidence } \\
\text { intervals }\end{array}$ & Yes & 368 & $\mathrm{NC}$ & $\mathrm{NC}$ & $\mathrm{NC}$ \\
\hline$[42]$ & No & Easy & No & $\begin{array}{l}\text { Just for a qualitative } \\
\text { estimation }\end{array}$ & Yes & 368 & $\mathrm{NC}$ & $\mathrm{NC}$ & $\mathrm{NC}$ \\
\hline$[43]$ & No & Easy & No & Considers population size & Yes & 368 & $\mathrm{NC}$ & $\mathrm{NC}$ & $\mathrm{NC}$ \\
\hline$[44]$ & Yes & Moderate & No & $\begin{array}{l}\text { Provides calculations just } \\
\text { for comparison of } \\
\text { quantitative outcomes }\end{array}$ & Yes & $\mathrm{NC}$ & $\mathrm{NC}$ & $\mathrm{NC}$ & $99+99$ \\
\hline$[45]$ & No & Advanced & No & $\begin{array}{l}\text { Provides all } 4 \text { types of } \\
\text { sample size calculations } \\
\text { Calculates confidence } \\
\text { intervals }\end{array}$ & No & 1475 & 171 & $212+212$ & $158+158$ \\
\hline$[46]$ & Yes & Advanced & No & $\begin{array}{l}\text { Provides all } 4 \text { types of } \\
\text { sample size calculations }\end{array}$ & No & 1475.12 & 170.73 & $\begin{array}{l}193.84 \\
\text { Total }\end{array}$ & $\begin{array}{l}223.25 \\
\text { Total } \\
\text { Considering } \\
\text { One SD }\end{array}$ \\
\hline$[47]$ & Yes & Easy & No & $\begin{array}{l}\text { Considers } \\
\text { population size }\end{array}$ & No & 368 & NC & NC & NC \\
\hline$[48]$ & No & Easy & No & $\begin{array}{l}\text { Only for } 50 \% \text { estimate } \\
\text { Considers } \\
\text { population size }\end{array}$ & No & 383 & $\mathrm{NC}$ & NC & NC \\
\hline$[49]$ & $\begin{array}{l}\text { Yes (Propor- } \\
\text { tions) } \\
\text { No } \\
\text { (Complex } \\
\text { Sample } \\
\text { Survey) }\end{array}$ & Easy & No & $\begin{array}{l}\text { Provides calculations for } \\
\text { complex sample surveys } \\
\text { Considers } \\
\text { population size }\end{array}$ & Yes & 368 & $\mathrm{NC}$ & $\mathrm{NC}$ & $\mathrm{NC}$ \\
\hline$[50]$ & No & Advanced & No & $\begin{array}{l}\text { Provides links to } \\
\text { JavaScript based forms for } \\
\text { simple power/sample size } \\
\text { calculations } \\
\text { (Comparing a Mean to a } \\
\text { Known Value, Comparing } \\
\text { Means for Two } \\
\text { Independent Samples, } \\
\text { Comparing a Proportion to a } \\
\text { Known Value, } \\
\text { Comparing } \\
\text { Proportions for Two } \\
\text { Independent } \\
\text { Samples, } \\
\text { Unmatched Case Control } \\
\text { Studies) }\end{array}$ & No & NC & NC & 97 & 112 \\
\hline
\end{tabular}


Meysamie et al. Journal of Medical Statistics and Informatics 2014,

continuation of Table 2.

\begin{tabular}{|c|c|c|c|c|c|c|c|c|c|}
\hline \multirow{3}{*}{ 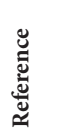 } & \multirow{3}{*}{$\begin{array}{l}\text { Providing } \\
\text { Formulas }\end{array}$} & \multirow[t]{3}{*}{ Usability } & \multirow{3}{*}{$\begin{array}{l}\text { Downloadable } \\
\text { File(s) }\end{array}$} & \multirow{3}{*}{$\begin{array}{l}\text { Type of Calculations } \\
\text { Provided }\end{array}$} & \multirow{3}{*}{$\begin{array}{l}\text { Accurate } \\
\text { calculation }^{\star}\end{array}$} & \multicolumn{4}{|c|}{ Common Sample Size Calculation Results } \\
\hline & & & & & & \multicolumn{2}{|l|}{ Estimation } & \multicolumn{2}{|l|}{ Comparison } \\
\hline & & & & & & Qualitative $^{1}$ & Quantitative $^{2}$ & Qualitative $^{3}$ & Quantitative $^{4}$ \\
\hline [51] & No & Advanced & No & $\begin{array}{l}\text { Only for } \\
\text { comparison of } 2 \text { means } \\
\text { Provides One and two sided } \\
\text { calculations } \\
\text { Considers single equal } \\
\text { variances } \\
\text { Calculates power }\end{array}$ & No & $\mathrm{NC}$ & $\mathrm{NC}$ & $\begin{array}{l}\text { One } \text { sided }=44 \\
\text { Two } \text { sided }=56\end{array}$ & $\mathrm{NC}$ \\
\hline$[52]$ & NR & NR & NR & $\begin{array}{l}\text { Offers different software for } \\
\text { sale } \\
\text { Sample Size \& Power } \\
\text { Analysis (nQuery } \\
\text { Advisor+nTerim) } \\
\text { Missing Data Analysis (Solas) } \\
\text { Analytical software (NCSS } \\
\text { Statistical Analysis and } \\
\text { Graphics Software) }\end{array}$ & NAP & $\mathrm{NC}$ & $\mathrm{NC}$ & $\mathrm{NC}$ & $\mathrm{NC}$ \\
\hline [53] & No & Easy & Yes & $\begin{array}{l}\text { Provides } \\
\text { calculations for estimations }\end{array}$ & Yes (just 2) & 367 & 42 & $\mathrm{NC}$ & $\mathrm{NC}$ \\
\hline$[54]$ & No & Easy & No & $\begin{array}{l}\text { Only for } 50 \% \text { estimate } \\
\text { Considers } \\
\text { population size } \\
\text { Just for } 95 \% \text { and } 99 \% \\
\text { confidence }\end{array}$ & No & 383 & $\mathrm{NC}$ & NC & $\mathrm{NC}$ \\
\hline [55] & No & Advanced & No & $\begin{array}{l}\text { Just calculates one sample for } \\
\text { Non parametric } \\
\text { survival }\end{array}$ & NAP & $\mathrm{NC}$ & $\mathrm{NC}$ & $\mathrm{NC}$ & $\mathrm{NC}$ \\
\hline$[56]$ & No & Easy & No & $\begin{array}{l}\text { Considers } \\
\text { response rate }\end{array}$ & Yes & 368 & $\mathrm{NC}$ & $\mathrm{NC}$ & $\mathrm{NC}$ \\
\hline [57] & No & Moderate & $\begin{array}{l}\text { Yes } \\
\text { (An excel file) }\end{array}$ & $\begin{array}{l}\text { Just calculates sample size for } \\
\text { complex sample survey }\end{array}$ & NAP & $\mathrm{NC}$ & $\mathrm{NC}$ & $\mathrm{NC}$ & $\mathrm{NC}$ \\
\hline$[58]$ & No & Advanced & $\begin{array}{l}\text { Yes } \\
\text { (A setup file) }\end{array}$ & $\begin{array}{l}\text { Calculates one tailed and two } \\
\text { tailed } \\
\text { Calculates for non equal groups } \\
\text { Calculates for paired groups } \\
\text { Calculates sample size for } \\
\text { correlation estimates } \\
\text { Considers default } 95 \% \text { power } \\
\text { Provides power analysis and } \\
\text { effect size } \\
\text { calculations } \\
\text { Provides calculations of P value } \\
\text { according to common statistical } \\
\text { tests } \\
\text { Draws power and sample size } \\
\text { plots }\end{array}$ & Yes (just 4) & $\mathrm{NC}$ & $\mathrm{NC}$ & $97+97$ & $100+100$ \\
\hline [59] & No & Advanced & Yes & $\begin{array}{l}\text { Provides sample size and power } \\
\text { calculations } \\
\text { Different equal and non equal } \\
\text { groups } \\
\text { Calculates one tailed and two } \\
\text { tailed }\end{array}$ & Yes (just 3) & $\mathrm{NC}$ & $\mathrm{NC}$ & $99+99$ & $107+107$ \\
\hline
\end{tabular}

1=Sample size calculation for estimating a qualitative variable with expected $40 \%$ prevalence and 5\% acceptable difference with $5 \%$ type I error.

$2=$ Sample size calculation for estimating a quantitative variable with expected SD as 10 and 3 units acceptable difference with $5 \%$ type I error. $3=$ Sample size calculation for comparison of a qualitative variable with expected $40 \%$ and $60 \%$ prevalence in groups with $5 \%$ type I error and $80 \%$ power.

$4=$ Sample size calculation for comparison of a quantitative variable with expected Mean \pm SD in groups as $25 \pm 7$ and $22 \pm 8$ with $5 \%$ type I error and $80 \%$ power.

${ }^{\star}$ In case of multiple calculations, all were not accurate, calculation numbers which was accurate (listed above) indicted in below parenthesis. 
and some uses variances instead of standard deviations for calculations [16] this can cause potential errors if researchers use standard deviations instead.

Some other sites provided advanced calculations for complex studies, $[12,26,49,55,57]$ in our study we did not rate and assess their calculations but when in simple ways of calculations there are so many faults how we can expect accurate results from those complex sites. Providing multi language support may be an ease of use for users with different languages other than English language. In our review we found two sites which provided multi language support $[23,37]$.

Some good references for reviewing sample size calculation principles are used in this study and can be helpful for researchers $[7,8]$. Special statistical software like STATA, SAS and $R$ also provides commands for sample size calculation and power analysis which can be useful for sophisticated approach.

\section{Conclusion}

It seems that those sites which provide calculating formulas can be more trusted for online sample size calculation and at least there will be a way for researchers to check the calculation accuracy based on the provided formulas. Although researchers who perform sophisticated studies need special assistance of statisticians for sample size calculation in their studies.

\section{Competing interests}

The authors declare that they have no competing interests.

Authors' contributions

\begin{tabular}{|l|c|c|c|c|c|c|}
\hline Authors' contributions & AM & FT & MMV & SYK & SEF & MA \\
\hline Research concept and design & $\checkmark$ & $\checkmark$ & -- & -- & -- & -- \\
\hline $\begin{array}{l}\text { Collection and/or assembly of } \\
\text { data }\end{array}$ & $\checkmark$ & $\checkmark$ & $\checkmark$ & $\checkmark$ & $\checkmark$ & -- \\
\hline Data analysis and interpretation & $\checkmark$ & $\checkmark$ & $\checkmark$ & $\checkmark$ & $\checkmark$ & $\checkmark$ \\
\hline Writing the article & $\checkmark$ & $\checkmark$ & $\checkmark$ & $\checkmark$ & $\checkmark$ & $\checkmark$ \\
\hline Critical revision of the article & $\checkmark$ & -- & -- & -- & -- & $\checkmark$ \\
\hline Final approval of article & $\checkmark$ & -- & -- & -- & -- & -- \\
\hline Statistical analysis & $\checkmark$ & $\checkmark$ & $\checkmark$ & $\checkmark$ & -- & -- \\
\hline
\end{tabular}

Acknowledgement

The authors thanks to Tehran University of Medical Sciences for providing the facilities to run an online sample size calculator and for supporting the study.

Publication history

Editor: Jimmy Efird, East Carolina University, USA. Received: 08-Jan-2013 Final Revised: 24-Feb-2014 Accepted: 26-Feb-2014 Published: 14-Apr-2014

\section{References}

1. Albert NM and O'Connor PC. How many subjects do I need in my research sample? Clin Nurse Spec. 2012; 26:302-4. | Article I PubMed

2. Schlesselman JJ. Sample size requirements in cohort and case-control studies of disease. Am J Epidemiol. 1974; 99:381-4. | Article I PubMed
3. Lwanga SK and Lemeshow S. Sample Size Determination in Health Studies: A Practical Manual, World Health Organization (1991). | Pdf

4. Mace AE. Sample-size determination. Reinhold, New York, 1964. | Book

5. Mellor B. Sample size methodology. M. M. Desu and D. Raghavarao, Academic Press, Boston, 1990. Statist Med. 1992; 11:562-563. | Article

6. Ahlbom A. Biostatistics for Epidemiologists. Lewis Publishers, Boca Raton, FL. 1993. I Website

7. Meysamie A and Peysepar R. Medical Statistics. Golbarg Press. 2012 (Persian).

8. Ryan TP. Sample size determination and power. John Wiley \& sons, USA. 1945. | Website

9. Donner A. Approaches to sample size estimation in the design of clinical trials--a review. Stat Med. 1984; 3:199-214. | Article | PubMed

10. 6sixsigma. | Website

11. Black berry world. | Website

12. Sample Size for Microarray Experiment. | Website

13. BOLD Educational Software Sample Size Calculator. | Website

14. DusickCalculating Sample Size. | Website

15. Easycalculation. | Website

16. Epi Tools (Ausvet animal health services). I Website

17. Statistical considerations for clinical trials and scientific experiments. | Website

18. Java applets for power and sample size. I Website

19. Advanced Sample Size Calculator. | Website

20. Tamaño de la muestra la calculadora. | Website

21. The research advisor. | Website

22. SampleSize The Research Advisors 2006. | Website

23. Online Sample Size Calculator. | Website

24. Power and Sample Size Calculation. I Website

25. Answers Research. | Website

26. Statistical Programs for Clinical Trials. | Website

27. Usability Sample Size Calculator. | Website

28. Sample Size Calculator (Calculator.net). I Website

29. Sample Size Calculator: Two Parallel-Sample Proportions. | Website

30. Calculate a survey sample size (number of respondents needed). । Website

31. Power/Sample Size Calculation for Logistic Regression with Binary Covariate(s). I Website

32. Evaluate your sample size using Decision Point's sample size calculator. (Decision point). I Website

33. Researcher's Toolkit, (DSS Research). | Website

34. Power and Sample Size Programs. | Website

35. Florida law firm. | Website

36. Sample Size Calculator. | Website

37. Solutions: Resources | Sample Size Calculator. | Website

38. IBP Strategy and Research. | Website

39. LCWA. I Website

40. MaCorr Research Solutions. I Website

41. National Statistical Services. | Website

42. Openuptomail. | Website

43. Pivotal Research Inc. | Website

44. SISA (simple interactive statistical analysis). I Website

45. Ques gen system inc. I Website

46. Sample Size Estimation. | Website

47. Raosoft Inc. | Website

48. Sample Size Calculator. I Website

49. Kevin's web page. | Website 
Meysamie et al. Journal of Medical Statistics and Informatics 2014,

http://www.hoajonline.com/journals/pdf/2053-7662-2-3.pdf

50. UCSF Biostatistics: Power and Sample Size Programs | Website

51. Power \& Sample Size Calculator. | Website

52. nQuery Advisor + nTerim. | Website

53. StatPac Statistics Calculator Sample Size Estimation. | Website

54. Creative research system. | Website

55. SWOG: Southwest Oncology Group. | Website

56. Torque Data. | Website

57. STEPS Sample Size Calculator and Sampling Spreadsheet, WHO. | Website

58. G*Power: Statistical Power Analyses for Windows and Mac. | Website

59. STATA. | Website

\section{Citation:}

Meysamie A, Taee F, Mohammadi-Vajari M-A, Yoosefi-Khanghah S, Emamzadeh-Fard S and Abbassi M. Sample size calculation on web, can we rely on the results? J Med Stat Inform. 2014; 2:3.

http://dx.doi.org/10.7243/2053-7662-2-3 